\title{
Measuring the invisible Higgs width at the 7 and 8 TeV LHC
}

\author{
Yang Bai, ${ }^{a}$ Patrick Draper $^{b}$ and Jessie Shelton ${ }^{c}$ \\ ${ }^{a}$ SLAC National Accelerator Laboratory, \\ 2575 Sand Hill Road, Menlo Park, CA 94025, U.S.A. \\ ${ }^{b}$ Santa Cruz Inst. for Particle Physics, Univ. of California, \\ Santa Cruz, CA 95064, U.S.A. \\ ${ }^{c}$ Department of Physics, Sloane Laboratory, Yale University, \\ New Haven, CT, 06520, U.S.A. \\ E-mail: poplarbai@gmail.com, patrickian@gmail.com, \\ jshelton137@gmail.com
}

ABSTRACT: The LHC is well on track toward the discovery or exclusion of a light Standard Model (SM)-like Higgs boson. Such a Higgs has a very small SM width and can easily have large branching fractions to physics beyond the SM, making Higgs decays an excellent opportunity to observe new physics. Decays into collider-invisible particles are particularly interesting as they are theoretically well motivated and relatively clean experimentally. In this work we estimate the potential of the 7 and $8 \mathrm{TeV}$ LHC to observe an invisible Higgs branching fraction. We analyze three channels that can be used to directly study the invisible Higgs branching ratio at the $7 \mathrm{TeV}$ LHC: an invisible Higgs produced in association with (i) a hard jet; (ii) a leptonic $Z$; and (iii) forward tagging jets. We find that the last channel, where the Higgs is produced via weak boson fusion, is the most sensitive, allowing branching fractions as small as $40 \%$ to be probed at 20 inverse fb for masses in the range between 120 and $170 \mathrm{GeV}$, including in particular the interesting region around $125 \mathrm{GeV}$. We provide an estimate of the $8 \mathrm{TeV}$ LHC sensitivity to an invisibly-decaying Higgs produced via weak boson fusion and find that the reach is comparable to but not better than the reach at the $7 \mathrm{TeV}$ LHC. We further estimate the discovery potential at the $8 \mathrm{TeV}$ LHC for cases where the Higgs has substantial branching fractions to both visible and invisible final states.

Keywords: Higgs Physics, Beyond Standard Model 


\section{Contents}

1 Introduction 1

2 Simple models for invisible Higgs decays 3

3 Direct limits from existing searches 4

3.1 Monojet searches 5

$\begin{array}{lll}3.2 & \text { Associated production of a Higgs with a } Z & 7\end{array}$

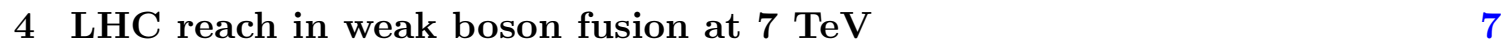

$\begin{array}{lll}4.1 & \text { LHC reach in weak boson fusion at } 8 \mathrm{TeV} & 12\end{array}$

5 Combination with SM Higgs search channels $\quad 14$

6 Conclusions and discussion $\quad 15$

\section{Introduction}

As the LHC approaches the first $10 \mathrm{fb}^{-1}$ of 7 and $8 \mathrm{TeV}$ data, we are entering a new era in Higgs physics. We will soon know whether or not a Standard Model (SM)-like Higgs boson exists. It becomes then an urgent and fascinating question to verify that any observed particle does - or does not — have the couplings and properties expected of the SM Higgs boson. Recent results presented by Atlas and CMS suggest a light SM-like Higgs may soon emerge from the data in the mass range below $130 \mathrm{GeV}[1,2]$. For such a Higgs boson, the smallness of the $b$ quark Yukawa coupling means that its total SM width is tiny: at $125 \mathrm{GeV}, \Gamma_{h}$ is only $4 \mathrm{MeV}$. Thus, a light SM-like Higgs boson is especially sensitive to the existence of physics beyond the Standard Model: even weak couplings to new light degrees of freedom can have an $\mathcal{O}(1)$ effect on the Higgs' branching ratios [3-8].

One particularly interesting candidate for new degrees of freedom into which the Higgs might decay is dark matter (DM) [9, 10]. Dark matter can couple to the SM through the Higgs portal [11, 12], which provides one of two leading interactions for physics in other sectors to couple to the SM. At colliders, Higgs-to-DM decays are invisible apart from large missing energy. The Higgs portal coupling also feeds into the Higgs-mediated DM-nuclear cross section, correlating the results of direct DM detection experiments with searches for invisible Higgs-to-DM decays at colliders. This correlation is not rigid, in the sense that a nonobservation in direct detection does not imply that the Higgs-to-DM branching ratio is small [10], but the two handles are complementary and can be used together to constrain models for DM (see, for example, [13-16]).

More generally, there are many compelling reasons to imagine that new light degrees of freedom exist and couple preferentially to the Higgs rather than to other parts of the 
SM. Several questions of naturalness in the Minimal Supersymmetric SM (MSSM) can be remedied by extending the MSSM Higgs sector with a singlet superfield [17]. Higgs decays into either the singlet or the singlino can then lead to cascade or invisible decays of the Higgs (for a review see [18]). Other examples which have been analyzed recently include decays of the Higgs through pseudo-Goldstone bosons to four jets [19], multiple SUSYbreaking sectors where the Higgs decays to two Goldstini [20], and a general analysis of Higgs descendants in [21]. In such scenarios, the Higgs is often produced with cross-sections close to the SM values, while the branching fractions to BSM particles can be large.

Searching for non-standard decay channels of a SM-like Higgs at the $7 \mathrm{TeV}$ LHC therefore is a flexible, generic, and extremely well-motivated probe of physics beyond the SM. It is also critical as a cross check of the SM Higgs production cross-sections: a universal discrepancy between the measured and expected Higgs cross-sections could be due either to suppression of the production cross-section from mixing with another degree of freedom, or suppression of the visible branching fractions by decay to invisible or buried [22, 23] final states. The tiny Higgs width in the low-mass region is too small to measure through its line shape, as might be imagined for a heavy Higgs [24]. The scenarios can only be distinguished by directly observing the nonstandard decay mode of the Higgs boson.

Existing searches for an invisibly-decaying Higgs at LEP [25] have set an upper bound on its mass at $95 \%$ confidence level (C.L.) of $114.4 \mathrm{GeV}$, assuming SM production and $100 \%$ Higgs decay into invisibles. At hadron colliders, an invisible Higgs signature is more difficult than at lepton colliders, as the inability to reconstruct events makes it challenging to separate the signal from the the large physics backgrounds coming from $Z$ (and $W$ ), which live at a similar mass scale as the signal. However, the invisible decay mode is still comparatively easy to detect. Large missing energy is a relatively clean signal of electroweak physics, cleaner than, for example, $b$-jets, and a signal in this channel may even be accessible in the $7 \mathrm{TeV}$ LHC run. Both theoretical [26-32] and experimental [3335 ] studies of an invisible Higgs at the $14 \mathrm{TeV}$ LHC have been performed, focusing on Higgs production from weak vector boson fusion (WBF) or in association with the $Z$ boson. Higgs production from gluon fusion plus one additional initial state radiation (ISR) jet has not been considered a promising channel at the LHC.

Our aim in the current work is to update these studies and estimate the sensitivity of the $7 \mathrm{TeV}$ and $8 \mathrm{TeV}$ LHC to an invisibly decaying Higgs. We first assess existing bounds on an invisible Higgs. The most stringent constraints are indirect, coming from the reinterpretation of SM Higgs exclusions as lower bounds on an invisible branching fraction, assuming SM Higgs production. There are also direct constraints arising from existing searches in both mono-jet $+E_{T}[36-38]$ and $h \rightarrow Z Z \rightarrow \ell^{+}+\ell^{-}+E_{T}[39,40]$, which we translate into bounds on the Higgs production times invisible branching fraction,

$$
\mathcal{B}_{\text {inv }} \equiv \sigma \times B R_{\text {inv }} / \sigma_{S M}
$$

These searches are sensitive to a light invisible Higgs, produced through $g g \rightarrow h j$ and $p p \rightarrow Z h$, respectively. We find that the mono-jet searches currently provide a better limit than the $Z+h$ channel, although a targeted optimization of the $Z+h$ channel might change this picture. 
Having established existing limits, we study the most sensitive channel for a light invisible Higgs, namely weak vector boson fusion, $q q \rightarrow h q q$. We perform a detailed detector-level study of the WBF channel to estimate the $7 \mathrm{TeV}$ and $8 \mathrm{TeV} \mathrm{LHC}$ reach. Incorporating improved strategies for controlling systematic errors, we find that invisible rates down to $\mathcal{B}_{\text {inv }} \approx 0.4$ can be excluded at $95 \% \mathrm{CL}$ with $20 \mathrm{fb}^{-1}$ at $7 \mathrm{TeV}$ and a similar reach is found at $8 \mathrm{TeV}$.

The layout of this paper is as follows. In section 2, we briefly discuss simple reference models which can give the Higgs an appreciable invisible branching fraction without altering electroweak symmetry breaking. The remainder of the paper is dedicated to estimating the sensitivity of the 7 and $8 \mathrm{TeV}$ LHC to an invisible Higgs decay mode. In sections 3.1 and 3.2 we adapt existing monojet $+E_{T}$ and $Z+E_{T}$ searches to establish current limits on $\mathcal{B}_{\text {inv }}$ from these channels. In section 4 we perform a detailed study of the weak boson fusion channel at the $7 \mathrm{TeV}$ LHC, which we find is the most sensitive channel. In section 4.1, we additionally estimate the sensitivity of the $8 \mathrm{TeV}$ LHC in this channel. In section 5 we compare the invisible search reach to the visible search reach and discuss the ability of the $8 \mathrm{TeV}$ LHC to constrain the hypothesis of an invisibly decaying Higgs through combinations of visible and invisible channels. Section 6 contains our conclusions.

\section{Simple models for invisible Higgs decays}

For simplicity, we consider a $\mathbb{Z}_{2}$ symmetry to protect one new particle beyond the standard model from decaying. This new particle could be a scalar $S$ (taken to be real) or a Dirac fermion $\chi$, which are SM singlets. Here we neglect the case of a new spin-one particle [41]. Starting from the scalar field $[9,15]$, we have the following potential,

$$
V(H, S)=-\mu^{2} H H^{\dagger}+\lambda\left(H H^{\dagger}\right)^{2}+\frac{1}{2} \mu_{S}^{2} S^{2}+\frac{1}{4} \lambda_{S} S^{4}+\kappa H H^{\dagger} S^{2} .
$$

After electroweak symmetry breaking $(\langle H\rangle=v / \sqrt{2}, v=246 \mathrm{GeV})$, the mass of the singlet becomes $m_{S}^{2}=\mu_{S}^{2}+\kappa v^{2}$, which should be positive to prevent a vacuum expectation value for $S$ and to keep the $\mathbb{Z}_{2}$ symmetry intact. The coupling modifying the Higgs decay is

$$
V(h, S)=\kappa v h S^{2} / 2+\cdots,
$$

which provides an invisible decay width for the Higgs boson,

$$
\Gamma(h \rightarrow S S)=\frac{\kappa^{2} v^{2}}{8 \pi m_{h}} \sqrt{1-\frac{4 m_{S}^{2}}{m_{h}^{2}}} .
$$

For a light Higgs boson, the dominant decay channel in the SM is into two $b$ quarks. Since the bottom Yukawa coupling is small, it is easy to modify the total width of Higgs boson and to suppress the SM visible decay branching ratios. We show the reduction of the visible branching ratios for two different Higgs masses $m_{h}=120,160 \mathrm{GeV}$ in figure 1 . We see from the left panel of figure 1 that the branching ratios through visible channels can be below $20 \%$ of the branching ratios in the pure SM. In this plot, we have chosen the bare scalar 

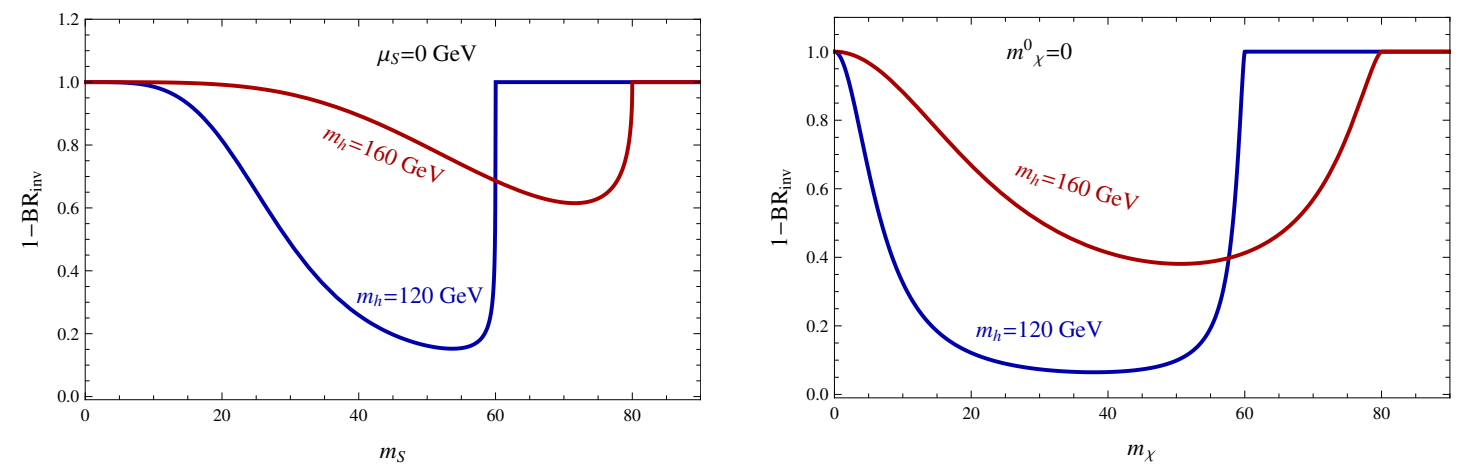

Figure 1. Left panel: the reduction of visible channel branching ratios after including a new invisible decay for the Higgs boson. The parameter $\mu_{S}$ is chosen to be zero, so the scalar $S$ has its mass proportional to the electroweak vacuum expectation value (VEV). Right: the same as the left panel but for a new fermion in the Higgs decay channel, with $m_{\chi}^{0}$ set to zero.

mass $\mu_{S}=0$ in eq. (2.1), so all of the $S$ mass comes from electroweak symmetry breaking, and we use the total SM widths calculated in ref. [42].

To generate Higgs decays to invisible fermions, we can extend the renormalizable model to include couplings

$$
\mathcal{L} \supset \lambda_{\chi} S \bar{\chi} \chi-2 \mu S H^{\dagger} H+m_{\chi}^{0} \bar{\chi} \chi
$$

We include a bare mass for $\chi$ for completeness, but let us assume for simplicity that $\lambda_{S}$ and $\kappa$ in eq. (2.1) (as well as any cubic coupling for $S$ ) are small. Then EWSB generates a VEV for $S,\langle S\rangle \approx \mu v^{2} / \mu_{S}^{2}$, and gives an additional mass contribution to $\chi$,

$$
m_{\chi}^{E}=\frac{\lambda_{\chi} \mu v^{2}}{\mu_{S}^{2}} .
$$

$S$ mixes with $h$, which allows $h$ to decay into $\bar{\chi} \chi$. The width is given by

$$
\Gamma(h \rightarrow \bar{\chi} \chi)=\frac{\left(m_{\chi}^{E}\right)^{2} m_{h}}{8 \pi v^{2}}\left(1-\frac{4 m_{\chi}^{2}}{m_{h}^{2}}\right)^{3 / 2} .
$$

where $m_{\chi}$ is the total mass for $\chi$. We show the reduction of visible channel branching ratios after including the $\bar{\chi} \chi$ invisible decay for the Higgs boson in the right panel of figure 1 .

\section{Direct limits from existing searches}

In this section we consider limits on an invisible Higgs from existing data, and briefly comment on future prospects in these channels. In section 3.1 we reinterpret searches in monojet $+E_{T}$ to establish sensitivity to an invisibly decaying Higgs produced through gluon fusion. In section 3.2 we reinterpret searches for $h \rightarrow Z Z \rightarrow \ell^{+} \ell^{-} \nu \bar{\nu}$ to obtain limits on an invisible Higgs produced in association with a $Z, p p \rightarrow h Z$. 

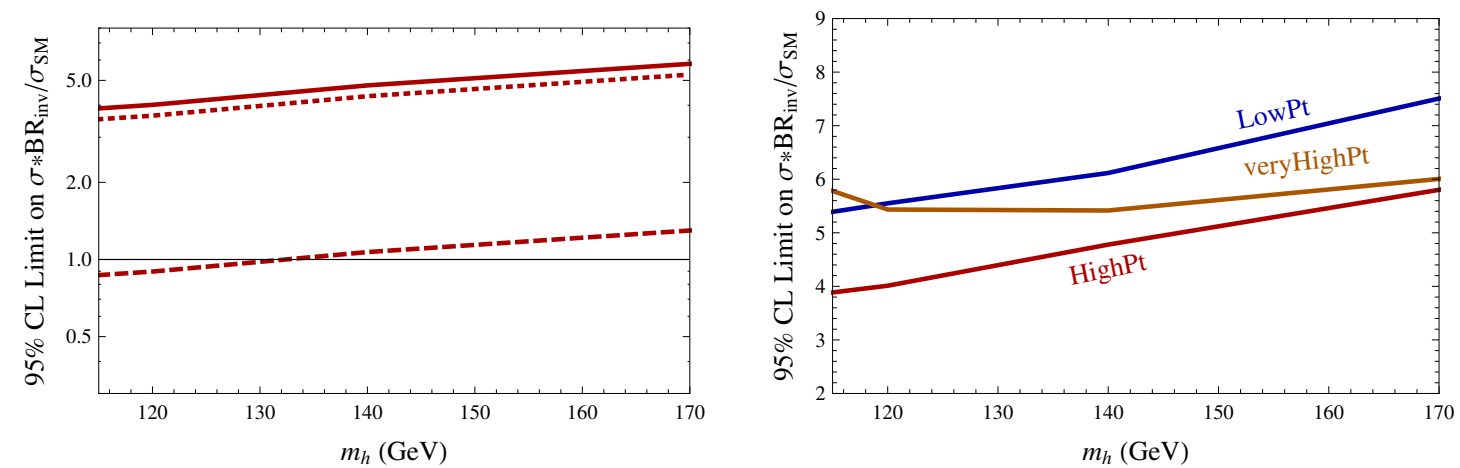

Figure 2. Left panel: the solid line is the current 95\% C.L. limit on the Higgs production times the invisible branching ratio from monojet searches at $1 \mathrm{fb}^{-1}$ with HighPt cuts. The dotted line is the projected limit at $20 \mathrm{fb}^{-1}$ assuming that the relative systematic error cannot be improved. The dashed line is the projected limit at $20 \mathrm{fb}^{-1}$ assuming that the relative systematic error can be improved and reduced alongside the statistical error by $1 / \sqrt{\mathcal{L}}$. Right panel: A comparison of limits from three different cuts used in ref. [38] with $1 \mathrm{fb}^{-1}$ luminosity.

\subsection{Monojet searches}

The simplest signal for invisible Higgs decays is monojet (or monophoton) events, made by producing a Higgs in the gluon fusion channel together with initial state radiation. Since the current monojet searches provide a more stringent constraint on the invisible Higgs rate $\mathcal{B}_{\text {inv }}$ than the monophoton searches, we will concentrate on the monojet signature and consider the existing searches constraining $\mathcal{B}_{\text {inv }}$.

Both CMS [36] and ATLAS [37, 38] have analyzed their monojet signatures at around $1 \mathrm{fb}^{-1}$ of integrated luminosity. In this section, we take the results from ATLAS [38] to set a constraint on $\mathcal{B}_{\text {inv }}$. Ref. [38] defines three sets of selection criteria with different $p_{T}$ thresholds demanded for the leading jet and the missing energy,

- LowPt: The leading jet is required to have $p_{T j_{1}}>120 \mathrm{GeV}$ and $\left|\eta_{j_{1}}\right|<2$, together with $\mathbb{E}_{T}>120 \mathrm{GeV}$. Other jets in the event $\left(\left|\eta_{j_{2}}\right|<4.5\right)$ must be sufficiently soft, $p_{T j_{2}}<30 \mathrm{GeV}$.

- HighPt: The leading jet is required to have $p_{T j_{1}}>250 \mathrm{GeV}$ and $\left|\eta_{j_{1}}\right|<2$, together with $E_{T}>220 \mathrm{GeV}$. Other jets in the event $\left(\left|\eta_{j_{2}}\right|<4.5\right)$ must be softer than $p_{T j_{2}}<$ $60 \mathrm{GeV}$ for the second-hardest jet, and $p_{T j_{3}}<30 \mathrm{GeV}$ for additional jets. The missing energy must be separated from the second-hardest jet in azimuth, $\Delta \phi\left(j_{2}, \mathbb{E}_{T}\right)<0.5$.

- veryHighPt: The leading jet is required to have $p_{T j_{1}}>350 \mathrm{GeV}$ and $\left|\eta_{j_{1}}\right|<2$, together with $\mathbb{E}_{T}>300 \mathrm{GeV}$. Other cuts on the subleading jets are the same as for the HighPt criteria.

In all cases, leptons are rejected for electrons (muons) above $p_{T}>20(10) \mathrm{GeV}$ and $|\eta|<$ $2.47(2.4)$.

We determine the efficiency of an invisibly decaying Higgs to pass these cuts to obtain current and projected limits coming from this channel. Signal events are generated using 
FeynRules [43] in conjunction with MadGraph5, and showered in PYTHIA [44]. We use PGS [45] to perform the fast detector simulation, after modifying the code to implement the anti- $k_{t}$ jet-finding algorithm. We generate our signal events $h+$ jets using MadGraph's native MLM matching [46] scheme and normalize to the inclusive production cross section of $h+$ jets at NNLO from ref. [47] to account for the large $K$-factor from next-to-leading order (NLO) and next-to-next-to-leading order (NNLO) corrections. In the present study, we neglect the contribution of signal events coming from weak boson fusion Higgs production, which contributes to the ATLAS monojet events at the $10 \%$ level.

In figure 2, the solid red line shows the existing $95 \%$ confidence level (C.L.) exclusion limit on $\mathcal{B}_{\text {inv }}$ from the mono-jet searches at ATLAS with a $1 \mathrm{fb}^{-1}$ luminosity. Using the HighPt cuts, we add the statistical and systematic errors in quadrature to set limits. To estimate the potential improvement with luminosity, we show with the dotted line the projected limits at $20 \mathrm{fb}^{-1}$ keeping the current relative systematic error intact. The dashed line shows the projected limits assuming that the relative systematic error can be reduced and scales as $1 / \sqrt{\mathcal{L}}$ as does the statistical uncertainty. In the right panel of figure 2 , we compare the sensitivities of the three different sets of cuts. As one can see from this panel, the HighPt cuts provide the best exclusion limit. ${ }^{1}$ This can be understood as follows: for the LowPt cuts the quoted systematic errors from multi-jets and non-collision backgrounds are enormous and limiting; on the other hand, for a sufficiently high $p_{T}$ cut, the major background $Z(\rightarrow \nu \bar{\nu})+$ jets has similar leading jet $p_{T}$ and $\mathbb{E}_{T}$ distributions as the signal, so increasing the cuts decreases the exclusion sensitivity.

Note that there is a large gap between the dotted line and the dashed line in figure 2, indicating the dominance of systematic errors in setting limits in this channel. The dashed line takes an optimistic view that all relative systematic errors can be scaled with luminosity as would be appropriate for uncertainties coming from data-driven methods. This is not an unreasonable approximation as the dominant uncertainties in ref. [38] are currently limited by control region statistics, but of course not all systematic errors will decrease with luminosity.

We conclude by pointing out that the invisible Higgs could be further separated from the dominant electroweak backgrounds by looking at the composition of the associated jets. Especially at lower $p_{T}$, the leading diagrams contributing to the signal are dominated by gluon radiation, while the $Z, W$ events are quark-enriched. Unfortunately the distinction is reduced at higher $p_{T}$, where the quark content is increased for both signal and background. However, since even at the LowPt working point electroweak processes are by far the dominant backgrounds, it is an interesting question whether additional sensitivity can be achieved by lowering the $p_{T}$ cuts of the HighPt and introducing quark-rejection variables [49]: the leading radiation in $h+j$ is $\sim 60 \%$ gluon-like at the LowPt working point, compared to $\sim 20 \%$ gluon-like for $Z$ and $W$. We consider this an interesting avenue for future work. However, we expect that this channel will have difficulty achieving the sensitivity offered by $h Z$ and WBF.

\footnotetext{
${ }^{1}$ ref. [48] has recently used the LowPt cuts to set a limit. We emphasize that the HighPt analysis is more sensitive and provides a better starting point for an experimental search for invisible Higgs decays.
} 


\subsection{Associated production of a Higgs with a $Z$}

We turn now to invisible Higgs decays in the so-called "Higgsstrahlung" channel, where the Higgs is produced in association with a $Z$ boson. One promising channel is $q \bar{q} \rightarrow Z+h \rightarrow$ $\ell^{+} \ell^{-}+\mathbb{E}_{T} .^{2}$ Although currently there is no invisible Higgs search in this channel at the LHC, the standard Higgs search in the channel $h \rightarrow Z Z \rightarrow \ell^{+} \ell^{-} \nu \bar{\nu}$ provides an identical final state and thus has the same backgrounds.

We consider the limits coming from the latest CMS search [40], which uses an integrated luminosity of $4.6 \mathrm{fb}^{-1}$. We summarize the set of cuts which offers the greatest sensitivity to a light invisible Higgs, namely the cuts used for $m_{h}=350 \mathrm{GeV}$ :

- Events must contain two opposite sign, same flavor leptons with $p_{T}>20 \mathrm{GeV}$ which reconstruct a $Z,\left|m_{Z}-m_{\ell \ell}\right|<15 \mathrm{GeV}$.

- The reconstructed $Z$ must have $p_{T}>55 \mathrm{GeV}$ and the missing energy must be greater than $\#_{T}>95 \mathrm{GeV}$.

- The transverse mass of an assumed $Z Z$ final state, $M_{T}^{2}=\left(\sqrt{p_{T, Z}^{2}+m_{Z}^{2}}+\sqrt{E_{T}^{2}+m_{Z}^{2}}\right)^{2}$ $-\left(\vec{p}_{T, Z}+\vec{\not}_{T}\right)^{2}$, should be in the range $298 \mathrm{GeV}<M_{T}<393 \mathrm{GeV}$.

- Events with $b$ jets with $p_{T, b}>30 \mathrm{GeV}$ and $\left|\eta_{b}\right|<2.4$ or additional leptons with $p_{T}>10 \mathrm{GeV}$ are vetoed, as are events containing a jet with $p_{T}>30 \mathrm{GeV}$ which is too close in azimuth to the missing energy, $\Delta \phi\left(j, \mathbb{E}_{T}\right)<0.5$. If the event contains no jets harder than $30 \mathrm{GeV}$, the azimuthal cut $\Delta \phi\left(j, \mathbb{E}_{T}\right)<0.5$ is applied to jets with $p_{T}>15 \mathrm{GeV}$.

We consider leptons within $|\eta|<2.5$.

We generate the signal events at tree-level using MadGraph5 and multiply the production cross section by a $K$-factor 1.3 from NNLO results [47]. After applying the CMS cuts on our signal events, we present the current constraint on $\mathcal{B}_{\text {inv }}$ in the solid line of figure 3. As one can see from figure 3, the invisible Higgs limit coming from this search is weaker than the limit coming from the monojet search.

These searches [39, 40] are not targeted to the invisible Higgs, and a dedicated analysis can improve the sensitivity; see ref. $[28,33,51]$ for studies at $14 \mathrm{TeV}$. However, we expect assisted production will not be as sensitive to an invisible Higgs as weak vector boson fusion, which we turn to next.

\section{LHC reach in weak boson fusion at $7 \mathrm{TeV}$}

The weak vector boson fusion (WBF) channel was previously shown to be the most sensitive channel to an invisible Higgs at the $14 \mathrm{TeV}$ LHC [27, 33, 34]. As the signal production proceeds mainly through the valence quark PDFs while the largest backgrounds are also

\footnotetext{
${ }^{2}$ We have also checked the $W+h \rightarrow \ell+\not T_{T}$ channel and found that the signal acceptance efficiency is very small using the cuts in ref. [50], and that the final exclusion limit is much worse than that from the $q \bar{q} \rightarrow Z+h \rightarrow \ell^{+} \ell^{-}+H_{T}$ channel; see also [51].
} 

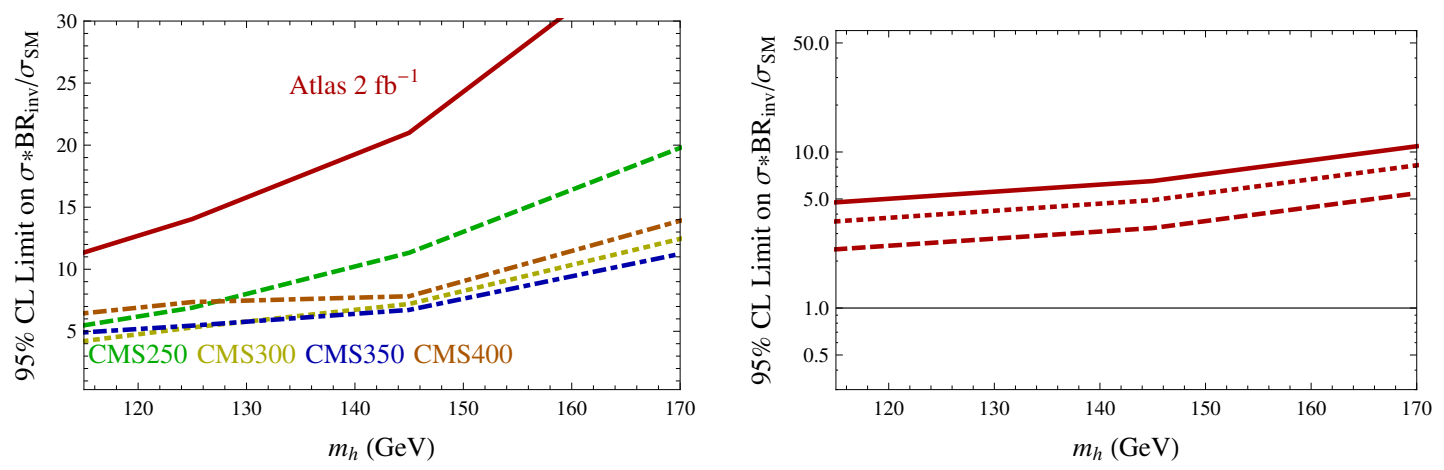

Figure 3. Left: comparison of limits on Higgs production times invisible branching ratio coming from different sets of cuts employed in [39, 40], interpreted as limits on $p p \rightarrow Z h \rightarrow \ell^{+} \ell^{-}+$invisibles. Right: the most stringent of the current limits and future projections. The solid line is the current 95\% C.L. limit from the CMS search at $4.6 \mathrm{fb}^{-1}$. The dotted line is the projected limit at $20 \mathrm{fb}^{-1}$ by assuming that the relative systematic error cannot be improved. The dashed line is the projected limit at $20 \mathrm{fb}^{-1}$ assuming that the relative systematic error can be improved and reduced alongside the statistical error by $1 / \sqrt{\mathcal{L}}$.

sensitive to $\bar{q}$ and $g$ PDFs, it is unsurprising that the WBF channel remains the most sensitive at $7 \mathrm{TeV}$. Here we perform a study of the $7 \mathrm{TeV}$ LHC's reach in this channel, which should dominate the LHC's reach during the low-energy run. The aim of this section is to comprehensively update the analysis of the WBF channel to $7 \mathrm{TeV}$, incorporating several advances in signal and background computations, and to assess the reach of the $7 \mathrm{TeV} \mathrm{LHC}$ for this signal. All results are detector-simulated using PGS.

The final state signature is characterized by two jets widely separated in rapidity together with large missing energy. The required missing energy is large enough that we anticipate this topology can be efficiently accepted through missing energy triggers. The major backgrounds are $Z \rightarrow \nu \bar{\nu}+$ jets from WBF; $W \rightarrow \ell \nu+$ jets from WBF, where the lepton is missed; $Z \rightarrow \nu \bar{\nu}+$ jets from QCD; $W \rightarrow \ell \nu+$ jets from QCD with a missing lepton; and finally mismeasured QCD.

To establish that we can neglect fake missing energy from QCD multi-jet events, we estimate the scale of the mismeasured multi-jet background by generating three-jet events using the Madgraph-Pythia pipeline and running them through PGS. Doing so, we find that the mismeasured three-jet background can be suppressed by more than an order of magnitude by demanding that the missing energy vector be sufficiently distant in $\phi$ from the closest identified jet,

$$
\operatorname{Min}\left(\Delta \phi_{j_{i}, \not_{T}}\right)>0.5
$$

The multi-jet contribution to the background falls off dramatically as a function of missing energy, and is entirely subdominant above $\not_{T} \gtrsim 100 \mathrm{GeV} .{ }^{3}$ While PGS only models the Gaussian portion of the detector response, the non-Gaussian tails will act to enhance configurations where a single mismeasured jet dominates the missing energy, thereby in-

\footnotetext{
${ }^{3}$ This accords with a similar conclusion obtained using Gaussian smearing in ref. [27]; see also fastdetector simulated experimental studies in [35].
} 
creasing the probability that the resulting event will fail the quality cut of eq. (4.1). Since after these two cuts the pure QCD background is orders of magnitude below the other backgrounds, we subsequently neglect this background.

The large production cross-section of the Higgs through gluon fusion means this contribution to the signal must be included, despite the low efficiency of this process to pass the selection cuts. We find that this channel contributes approximately $10 \%$ of the signal after all selection cuts, broadly in accord with studies at $14 \mathrm{TeV}$ [52-54].

The signal events as well as the physics backgrounds are generated in MadGraph5 and showered in PYTHIA. Non-WBF processes where the jets arise from QCD radiation $(g g \rightarrow h+$ jets, $Z+$ jets, $W+$ jets $)$ are matched out to two jets using Madgraph's native MLM matching scheme. Detector simulation is done in PGS using $R=0.5$ anti- $k_{T}$ jets. After establishing our selection cuts, we have checked that the overall efficiencies for leading order $(W \rightarrow \tau \nu)+2 j$ events are similar to those for fully matched $(W \rightarrow \tau \nu)+$ jets events, and to increase our Monte Carlo statistics we supplement our matched $(W \rightarrow \tau \nu)+$ jets backgrounds with a sample of fixed $(W \rightarrow \tau \nu)+2 j$ events.

Signal events produced through gluon fusion, $g g \rightarrow h$, are normalized to the inclusive NLO production cross-sections tabulated in [55]. Signal events produced through WBF are multiplied by a constant $K$-factor of 0.95 (for all values of $m_{h}$ ) which accounts for both QCD and electroweak corrections [56]. We use VBFNLO [57] to obtain a constant $K$-factor of 1.1 for the WBF $p p \rightarrow Z j j, p p \rightarrow W j j$ backgrounds. The $W+$ jets cross-section is normalized to the inclusive cross-section of [58]. Numerically this is very close to using the LO cross-section for $W+2 j$ from Madgraph and applying the $K$-factor corresponding to the ratio of leading order and next-to-leading order $W+2 j$ cross-sections as found in [58]. Correspondingly, we normalize the the $Z+$ jets cross-section by multiplying the LO Madgraph cross-section with the ratio of leading order and next-to-leading order $Z+2 j$ cross-sections found in [59].

To estimate the $W$ backgrounds, we assume that events with sufficiently hard and central leptons can be rejected. Specifically, if an event contains a truth-level lepton with $\left|\eta_{\ell}\right|<2.5$, we assume the event can be rejected if the lepton $p_{T}$ satisfies $p_{T}>20 \mathrm{GeV}$ for electrons, $p_{T}>10 \mathrm{GeV}$ for muons, and $p_{T}>20 \mathrm{GeV}$ for visible hadronic taus. The resulting signal and background cross-sections are displayed in table 1 for an initial set of reference cuts, namely

$$
\not_{T}>90 \mathrm{GeV} \quad \text { at least } 2 \text { jets with } p_{T j}>20 \mathrm{GeV} \quad \operatorname{Min}\left(\Delta \phi_{j_{i}, \not_{T}}\right)>0.5
$$

in addition to the lepton veto.

Our main selection cuts are:

$E_{T}>120 \mathrm{GeV} \quad$ at least 2 jets with $p_{T j}>30 \mathrm{GeV}, \quad M_{j_{1} j_{2}}>1200 \mathrm{GeV}, \quad \Delta \eta_{j_{1} j_{2}}>4.5$.

We also cut on the azimuthal angle of the tagging jets, requiring that they not be back-to-back:

$$
\Delta \phi_{j_{1} j_{2}}<1.5
$$




\begin{tabular}{|c|c|c|c|c|c|c|}
\hline Cross section $(\mathrm{fb})$ & $q q h$ & $h j j$ & $q q Z$ & $Z j j$ & $q q W$ & $W j j$ \\
\hline Reference cuts & 315 & 647 & 404 & 32600 & 465 & 32300 \\
\hline WBF selection cuts & 14.1 & 1.86 & 6.82 & 24.8 & 7.30 & 17.9 \\
$\Delta \phi$ cut & 9.26 & 1.39 & 2.05 & 12.5 & 2.53 & 9.73 \\
jet veto (1) & 4.23 & 0.41 & 0.77 & 3.37 & 1.11 & 2.89 \\
jet veto (2) & 7.25 & 0.67 & 1.23 & 7.58 & 1.81 & 6.08 \\
\hline
\end{tabular}

Table 1. Cross-sections in fb for signal and background in the vector boson fusion Higgs search channel. Signal cross-sections are shown for $m_{h}=120 \mathrm{GeV}$. The cuts are described in the text.

The azimuthal angle is the one distribution which has a markedly different shape for the Higgs than for other WBF processes [27]. As this distribution probes the spin correlations of the jets, it is not modeled faithfully by jets arising from parton showers in Pythia. To model the efficiency for background $W, Z+$ jets processes to pass the $\Delta \phi$ cut, we consider fixed order $W, Z+2$ jet events generated in MadGraph, which does retain spin information. We find that the difference in efficiency between fixed order and matched events decreases as $m_{j j}$ increases, and is order $10 \%$ in the regions of interest here. Accordingly, we account for the lack of spin correlations in the matched samples by multiplying the efficiencies for $W, Z+$ jets to pass the $\Delta \phi$ cut by a factor of 1.1 .

Lastly, we impose a central jet veto. We show results for two different implementations of the veto,

1. requiring any central jet $(|\eta|<2.5)$, including the two tagging jets, to have $p_{T}<$ $40 \mathrm{GeV}$, and

2. requiring any central jet in addition to the two leading jets to have $p_{T}<25 \mathrm{GeV}$.

The second and more traditional implementation of the jet veto offers a better limit with only statistical uncertainties taken into account, but the first implementation of the veto gives the best results after systematic uncertainties are included according to our prescription. Signal and background cross-sections through these cuts are displayed in table 1.

Note that $W+$ jets with a missing lepton, and in particular with a missing $\tau$, is numerically as large as the background from the $Z$. In particular $W \rightarrow \tau \nu+$ jets contributes more than half of the $W$ background. Vetoing more aggressively on charged leptons is thus one obvious avenue to maximize the sensitivity. Another possible avenue of improvement is lowering the missing energy cut, which at $120 \mathrm{GeV}$ is slightly too hard for optimal significance. We keep the missing energy cut hard to be conservative about trigger acceptance, forward jet resolution, and multi-jet background, but lowering this threshold could well prove useful in this channel.

We plot the estimated 95\% CL exclusion reach of the $7 \mathrm{TeV}$ LHC in the left panel of figure 4. Since no Higgs mass peak can be reconstructed, this signal is purely a counting experiment, and systematic uncertainties on the background cannot be neglected when estimating the reach in this channel. To reduce theoretical uncertainties associated with 

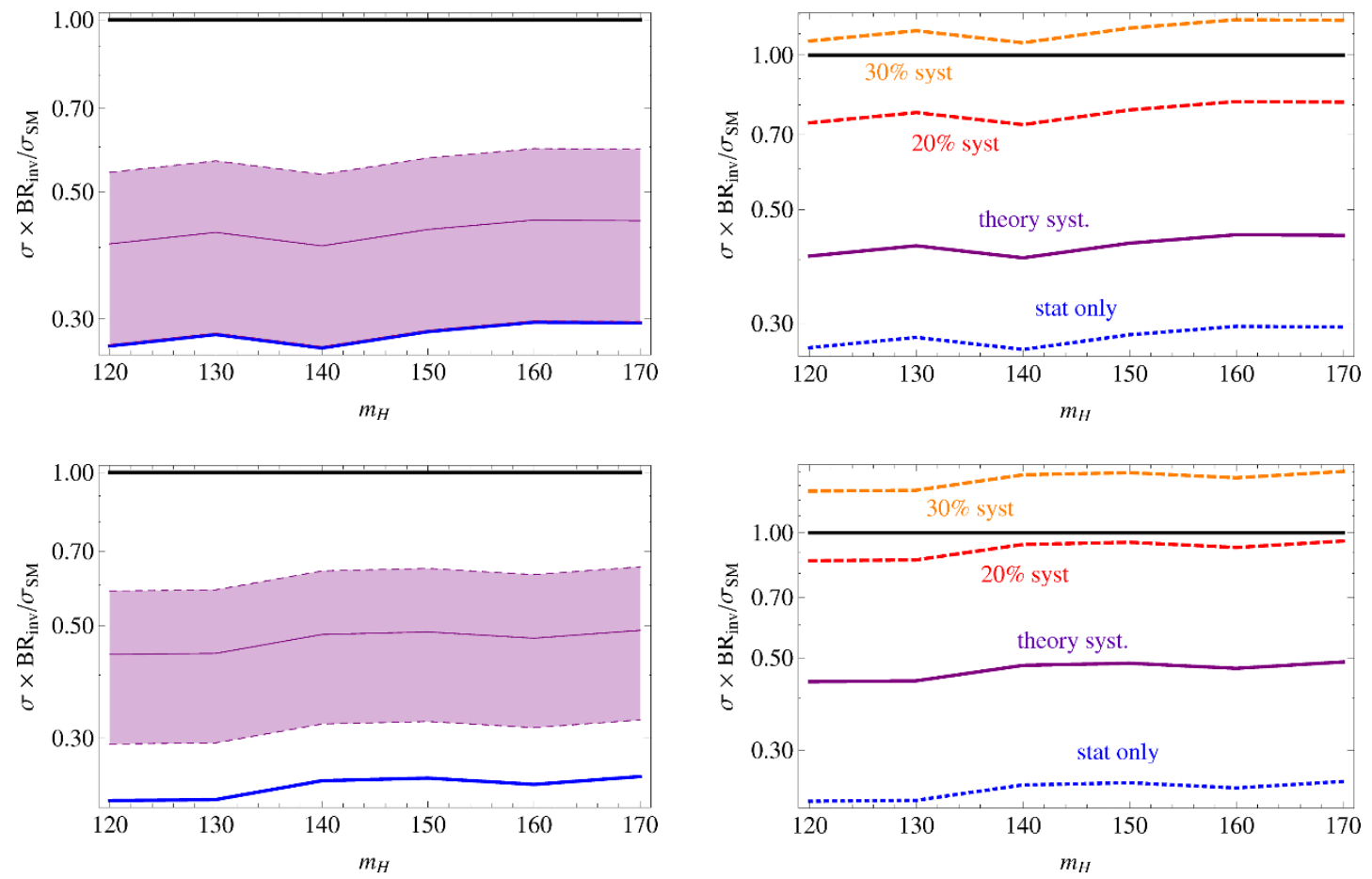

Figure 4. Left: estimated $95 \%$ CL limit on the invisible branching fraction of the Higgs with $20 \mathrm{fb}^{-1}$ of data at the $7 \mathrm{TeV}$ LHC. The straight line at 1 denotes a Higgs produced with SM cross-sections decaying entirely into invisibles. Shaded bands show $\pm 1 \sigma$ with systematic uncertainties assigned as described in the text. Right: dependence of the $95 \%$ CL limits on systematic uncertainties. The top row shows results for jet veto (1) as described in the text; the bottom row shows results for jet veto $(2)$.

modeling the $Z, W+$ jets backgrounds, the backgrounds can be modeled from control regions in the data. Inverting the lepton selection criteria for $W+$ jets furnishes a large control sample. For $Z+$ jets, the control sample provided by $Z \rightarrow \ell^{+} \ell^{-}$is statistically limited by the small leptonic branching ratio of the $Z$. A more promising technique is to reweight $\gamma+$ jets, exploiting the stability of the ratio $\sigma(\gamma+X) / \sigma(Z+X)$ [60-62]. We expect that we are in the regime where this technique can be applied. The cut of eq. (4.1) requiring the missing energy to be azimuthally separated from the jets ensures that we do not probe the region of phase space where the photon amplitudes are dominated by the collinear singularity, and the missing energy cut we apply is not substantially different from those studied in [62]. Using photons allows the $Z \rightarrow \nu \bar{\nu}$ contribution to the background to be determined to below $10 \%$. We assume a $10 \%$ theoretical uncertainty on $W, Z+$ jets when calculating our systematic error bars. The WBF production cross-sections for $W, Z$ are relatively stable [63] and we assume a 5\% theoretical uncertainty for these backgrounds.

The cuts employed in this analysis do not exploit all of the kinematic information in the final state: the azimuthal distance between the missing energy and the jets contains some distinguishing power between signal and background. We have checked that cutting on appropriate linear combinations of $\Delta \phi_{E_{T} j_{1}}$ and $\Delta \phi_{j_{1} j_{2}}$ offers a marginal improvement 
in the reach. However, the azimuthal angle of the missing energy is subject to larger experimental systematic uncertainties than the (well-measured) azimuthal angles of the jets, so it is unclear whether the improvement will persist after all experimental systematic uncertainties have been taken into account. We comment that since the signal process in this channel is relatively well described by a LO matrix element, a matrix element technique $[64,65]$ seems promising as a way to fully exploit the event kinematics and offer improved separation between signal and background. A fully accurate estimate of the $7 \mathrm{TeV}$ LHC's reach in this channel however requires a more accurate detector simulation. In particular, the final state signature is subject to uncertainties in the forward jet energy scale which are exacerbated by pileup. Large experimental systematics on jet resolution could spoil the sensitivity of a matrix element-based kinematic fitter. The right panel of figure 4 shows how the estimated limit weakens as larger systematic uncertainties are included. We consider the results in this section strong motivation for a more detailed investigation of this channel.

\subsection{LHC reach in weak boson fusion at $8 \mathrm{TeV}$}

NLO calculations are not available at $8 \mathrm{TeV}$ for all of the required signal and background processes. Nonetheless, we can estimate the reach of the $8 \mathrm{TeV}$ LHC by taking the $K$-factors to be approximately equal at $7 \mathrm{TeV}$ and at $8 \mathrm{TeV}$. We generate signal and background WBF processes at $\mathrm{LO}$ and normalize with the same constant $K$-factor used at $7 \mathrm{TeV}$. We generate matched $W, Z, h+$ jets samples at $8 \mathrm{TeV}$, and normalize to the $7 \mathrm{TeV}$ NLO cross-sections, then rescale by the ratio $\Delta=\sigma_{i n c, 8}^{(0)} / \sigma_{i n c, 7}^{(0)}$.

The resulting signal and background cross-sections are displayed in table 1 for an initial set of reference cuts, namely

$$
\ddot{E}_{T}>90 \mathrm{GeV}, \quad \text { at least } 2 \text { jets with } p_{T j}>20 \mathrm{GeV}, \quad \operatorname{Min}\left(\Delta \phi_{j_{i}, \not_{T}}\right)>0.5,
$$

in addition to the lepton veto.

At $8 \mathrm{TeV}$, the selection cuts can be tightened:

$$
\not_{T}>120 \mathrm{GeV} \text { at least } 2 \text { jets with } p_{T j}>35 \mathrm{GeV}, \quad M_{j_{1} j_{2}}>1600 \mathrm{GeV}, \Delta \eta_{j_{1} j_{2}}>4.5,
$$

in addition to the cut on the azimuthal angle of the tagging jets,

$$
\Delta \phi_{j_{1} j_{2}}<1.5 \text {. }
$$

We again apply a correction factor of 1.1 to the $W, Z+$ jets backgrounds after the $\Delta \phi$ cut to better model the angular distributions. Finally, we apply a central jet veto, requiring no additional central jets with $p_{T}<20 \mathrm{GeV}$. Signal and background cross-sections through these cuts are displayed in table 2 .

Prospects at the $8 \mathrm{TeV}$ LHC do not improve relative to the $7 \mathrm{TeV}$ LHC for the same amount of integrated luminosity. This is unsurprising: the signal, which arises dominantly from valence quark PDFs, does not increase as quickly with $\sqrt{s}$ as do the background $W$ and $Z+$ jets processes. Tightening the selection cuts to improve $S / \sqrt{B}$ further suppresses the contribution of $g g \rightarrow h j j$ to the signal reach. Thus in this channel the increased center of mass energy gives little advantage. 


\begin{tabular}{|c|c|c|c|c|c|c|}
\hline Cross section (fb) & $q q h$ & $h j j$ & $q q Z$ & $Z j j$ & $q q W$ & $W j j$ \\
\hline Reference cuts & 404 & 951 & 517 & 39100 & 594 & 49400 \\
\hline WBF selection cuts & 14.1 & 1.86 & 6.82 & 24.8 & 7.30 & 17.9 \\
$\Delta \phi$ cut & 5.72 & 0.61 & 1.65 & 3.73 & 1.68 & 6.89 \\
jet veto & 4.11 & 0.32 & 1.27 & 2.40 & 1.30 & 4.20 \\
\hline
\end{tabular}

Table 2. $8 \mathrm{TeV}$ cross-sections in fb for signal and background in the weak boson fusion Higgs search channel. Signal cross-sections are shown for $m_{h}=120 \mathrm{GeV}$. The cuts are described in the text.
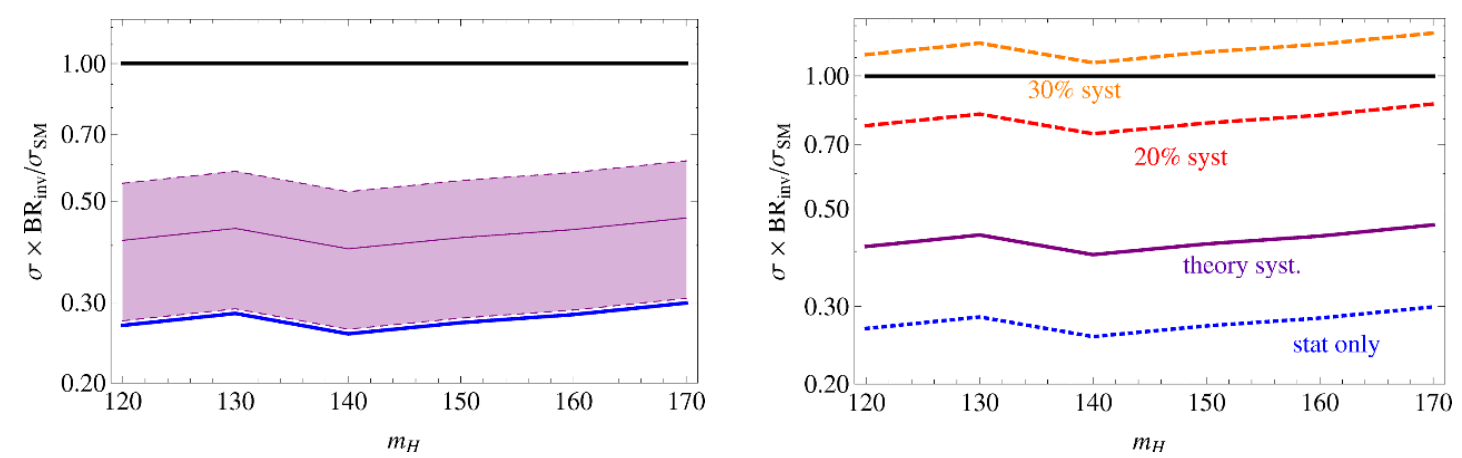

Figure 5. Left: Estimated 95\% CL limit on the invisible branching fraction of the Higgs with $20 \mathrm{fb}^{-1}$ of data at the $8 \mathrm{TeV}$ LHC. The straight line at 1 denotes a Higgs produced with SM crosssections decaying entirely into invisibles. Shaded bands show $\pm 1 \sigma$ with systematic uncertainties assigned as described in the text. Right: Dependence of the 95\% CL limits on systematic uncertainties.
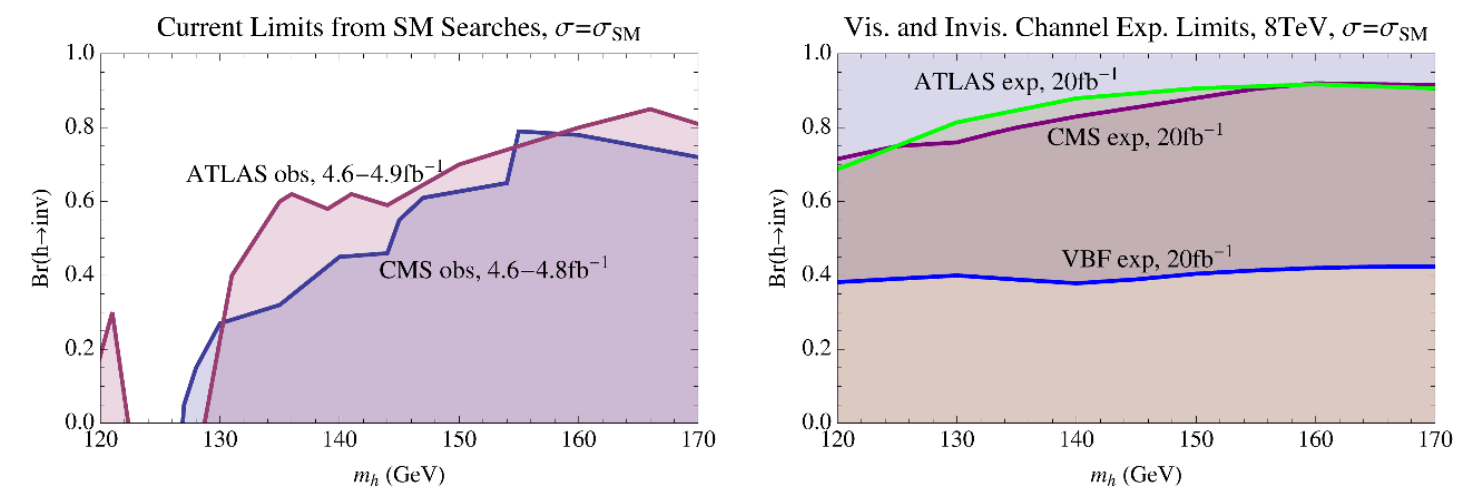

Figure 6. Left: present observed lower bound on the invisible branching ratio assuming the IHH. Right: expected lower limit from visible channels and upper limit from the WBF invisible channel with $20 \mathrm{fb}^{-1} /$ channel at $8 \mathrm{TeV}$. 


\section{Combination with SM Higgs search channels}

If no signal is observed in SM Higgs channels, it will be of interest to analyze the channels probing invisible Higgs decays. We define the invisible Higgs hypothesis (IHH) to be the case $\left\{\sigma=\sigma_{S M}, B R(h \rightarrow\right.$ inv $)+B R(h \rightarrow$ vis $\left.)=1\right\}$, or $\mathcal{B}_{\text {inv }}=B R(h \rightarrow$ inv $)$. Current SM Higgs limits can then be reinterpreted as lower limits on $B R(h \rightarrow$ inv $)$ in the IHH, as shown in the left-hand panel of figure 6 .

If no signal is observed in the invisible channels, then upper limits may be placed on the IHH, providing complementary coverage as shown in the right-hand panel of figure 6 for $20 \mathrm{fb}^{-1} /$ channel at $8 \mathrm{TeV}$ (projections for visible channels are made with $\sqrt{\mathcal{L}}$ rescaling of the expected limits given in $[66,67]$.) For sufficiently large invisible widths, the total Higgs width can begin to exceed the experimental resolution of the Higgs bump, which would weaken the limits from the visible channels. This effect is not large, and negligible in the very light $\left(m_{h} \lesssim 130 \mathrm{GeV}\right)$ range of most interest. We use the expected limits from the visible channel searches directly without modification.

Even better limits may be derived by combining the visible and invisible SM Higgs searches. Allowing the cross section to float, one can make the usual "Brazil-band" plots, where now the $y$-axis parametrizes a space of models that differ from the IHH by a universal rescaling $R$ of each channel's rate. Limits below $R=1$ exclude the IHH for a given pair $\left\{m_{h}, B R(h \rightarrow \mathrm{inv})\right\}$. Strong limits will motivate a variety of interpretations not covered by the IHH - perhaps there is no Higgs, or the Higgs is buried, or the production cross section is suppressed. Values of $R<1$ can be interpreted as a minimum required universal suppression factor of the cross section, or one minus the minimum required branching ratio into "buried" final states.

We can make estimates for the combined reach of SM+invisible search channels with a quadrature combination of the statistical significances, or inverse quadrature combination of the individual limits on $R$,

$$
\left(\frac{1}{R^{95}}\right)^{2} \approx\left(\frac{1}{R_{\mathrm{vis}}^{95}}\right)^{2}+\left(\frac{1}{R_{\mathrm{inv}}^{95}}\right)^{2} .
$$

Here $R^{95}$ denotes the $95 \%$ CL limit on $R$. Since the error bars in each channel include systematic errors that are in general correlated across channels, our combinations are approximate and intended only to suggest the potential interplay between visible and invisible channels. To estimate the search sensitivity to the visible Higgs decay modes, we use the recent expected limits from CMS [1] as they combine several channels with (almost) uniform luminosity per channel.

The expected limits obtained from a combination of CMS visible channels and the WBF invisible channel at $20 \mathrm{fb}^{-1} /$ channel and $8 \mathrm{TeV}$ are shown in the right panel of figure 7 as a function of $m_{h}$ and $B R(h \rightarrow$ inv $)$. We use only the WBF channel; in principle the reach could be extended using monojet and in particular $h Z$ in combination. The left panel of figure 7 shows the reach for $B R(h \rightarrow$ inv $)=0.5$ without channel combination, demonstrating the increase in sensitivity afforded by the combination, particularly at low Higgs masses. 

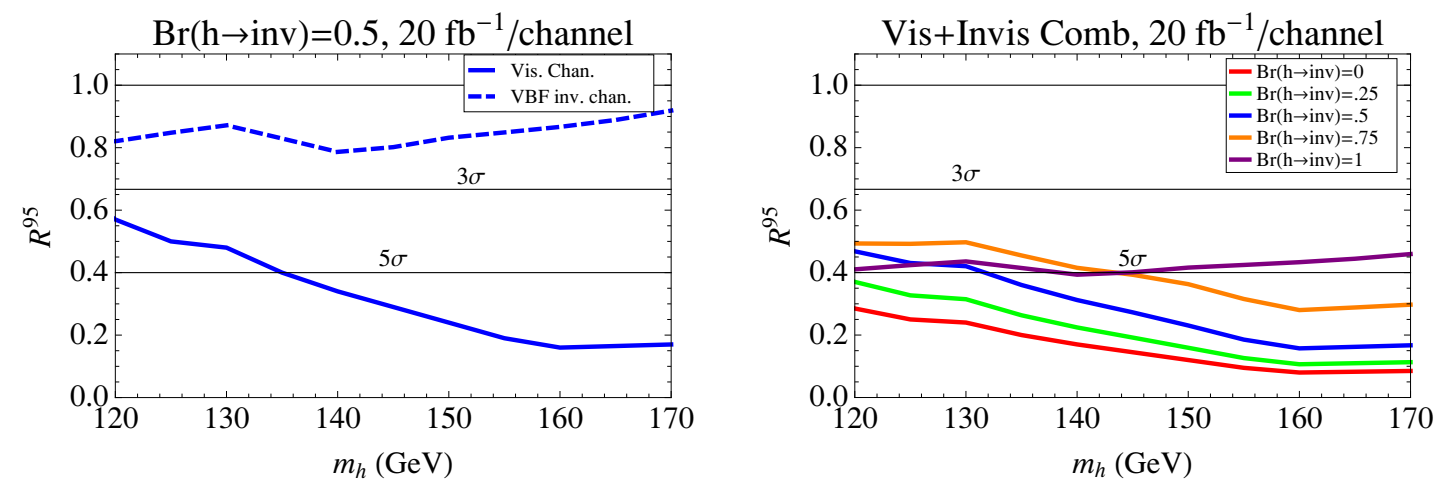

Figure 7. Left: $8 \mathrm{TeV}$ expected CMS visible and WBF invisible 95\% CL reaches for the IHH with $50 \%$ branching to invisibles. Right: $8 \mathrm{TeV}$ expected $\mathrm{CMS}$ visible+WBF invisible combined reach for several $h \rightarrow$ invisible branching fractions. Horizontal lines indicate where sensitivity reaches 3 and $5 \sigma$; curves below the lines indicate models that can either be strongly excluded, or would be expected to give enough signal for evidence or observation.

For low Higgs masses, the invisible reach is increasingly comparable to the visible channels. If the expected reach in invisible channels with $B R(h \rightarrow$ inv $)=1$ is comparable to the expected reach in visible channels with $B R(h \rightarrow$ vis $)=1$, then the combined expected reach for mixed branching ratios is weaker - for this reason $B R(h \rightarrow$ inv $)=0.5$ at $m_{h}=120 \mathrm{GeV}$ has lower significance than either $B R(h \rightarrow$ inv $)=0$ or $B R(h \rightarrow$ inv $)=1$ at the same mass.

Similarly, if a signal is seen in the SM Higgs search channels, but the best-fit rate is less than expected from the SM, the invisible channels will be relevant. If a signal is observed in these channels, then an SM+invisible combination becomes very interesting; if no invisible signal is detected then this suggests either altered production cross-sections or different beyond-the-SM decay modes.

\section{Conclusions and discussion}

If a Higgs-like particle is observed at the LHC, measuring its properties in detail is important for understanding the emerging description of electroweak symmetry breaking. New physics beyond the Standard Model can distort the Higgs production cross-sections and branching fractions. The branching fractions of a light Higgs $\left(m_{h} \lesssim 2 m_{W}\right.$, and in particular $m_{h} \sim 125 \mathrm{GeV}$ ) are particularly sensitive to the existence of new light degrees of freedom, owing to the small SM width of a Higgs below the $W$ threshold. Searching for beyond-theSM decays of a Higgs boson is thus an important cross check of Higgs properties.

We have focused here on light Higgs decay into invisible particles, as (1) these decays are theoretically well motivated by the existence of dark matter among other considerations, and (2) the signatures of these decays are relatively clean, allowing sensitivity to branching fractions $\mathcal{B}_{\text {inv }}=\sigma \times B R(h \rightarrow$ inv $) / \sigma_{S M}<1$ already at the 7 and $8 \mathrm{TeV}$ LHC. We have studied the 7 and $8 \mathrm{TeV}$ LHC reach for invisibly decaying Higgs bosons. We have obtained the current direct upper limits on $\mathcal{B}_{\text {inv }}$ from experimental searches in $j+\mathbb{E}_{T}$ and 
$\ell^{+} \ell^{-}+E_{T}$ final states, and performed a full detector-level study of the weak vector boson fusion channel, which is the most sensitive. Our analysis of the WBF channel is the first study at 7 and $8 \mathrm{TeV}$ for the signal $h \rightarrow$ invisibles. We find, after inclusion of the $g g \rightarrow h+$ jets contribution to the signal and arguing for the use of reweighted $\gamma+$ jets to reduce the systematic errors in the $Z, W+$ jets backgrounds, that invisible Higgs branching fractions as low as $\mathcal{B}_{\text {inv }} \gtrsim 0.4$ can be excluded with $20 \mathrm{fb}^{-1}$ of data at 7 and $8 \mathrm{TeV}$ for all Higgs masses below $170 \mathrm{GeV}$.

Finally, we have considered the complementarity between searches in visible and invisible channels. If the branching ratios are highly mixed, then the hypothesis of invisible Higgs decays (as opposed to direct suppression of the Higgs production cross section) can be efficiently tested by a combination of the two types of channels. The interesting question of establishing whether Higgs production and decay are indeed SM-like is thus one that the LHC can begin to usefully address even in the low-energy run.

\section{Acknowledgments}

We would like to thank H. Beauchemin, M. Buckley, L. Dixon, T. Golling, and S. Hoeche for useful discussions and comments, and A. Hook for providing modified PGS code. SLAC is operated by Stanford University for the US Department of Energy under contract DEAC02-76SF00515. PD was supported by DOE grant DE-FG02-04ER41286. JS was supported by the DOE grant DE-FG02-92ER40704 and by the LHC Theory Initiative through the grant NSF-PHY-0969510. JS and YB thank the KITP where this work was initiated and which receives support from the National Science Foundation under Grant No. NSF PHY05-51164. This work was also supported by the facilities and staff of the Yale High Performance Computing Center through the NSF grant CNS 08-21132.

Open Access. This article is distributed under the terms of the Creative Commons Attribution License which permits any use, distribution and reproduction in any medium, provided the original author(s) and source are credited.

\section{References}

[1] CMS collaboration, Combination of SM Higgs searches, PAS-HIG-11-032 (2011).

[2] ATLAS collaboration, Combination of Higgs Boson Searches with up to $4.9 \mathrm{fb}^{-1}$ of pp collisions data taken at a center-of-mass energy of $7 \mathrm{TeV}$ with the ATLAS experiment at the LHC, ATLAS-CONF-2011-163 (2011).

[3] R.E. Shrock and M. Suzuki, Invisible decays of Higgs bosons, Phys. Lett. B 110 (1982) 250 [INSPIRE].

[4] J.R. Ellis, J. Gunion, H.E. Haber, L. Roszkowski and F. Zwirner, Higgs bosons in a nonminimal supersymmetric model, Phys. Rev. D 39 (1989) 844 [INSPIRE].

[5] U. Ellwanger, J.F. Gunion and C. Hugonie, Establishing a no lose theorem for NMSSM Higgs boson discovery at the LHC, hep-ph/0111179 [INSPIRE].

[6] U. Ellwanger, J. Gunion, C. Hugonie and S. Moretti, NMSSM Higgs discovery at the LHC, hep-ph/0401228 [INSPIRE]. 
[7] D. O'Connell, M.J. Ramsey-Musolf and M.B. Wise, Minimal extension of the standard model scalar sector, Phys. Rev. D 75 (2007) 037701 [hep-ph/0611014] [INSPIRE].

[8] W.-Y. Keung and P. Schwaller, Long lived fourth generation and the Higgs, JHEP 06 (2011) 054 [arXiv: 1103.3765] [inSPIRE].

[9] C. Burgess, M. Pospelov and T. ter Veldhuis, The minimal model of nonbaryonic dark matter: a singlet scalar, Nucl. Phys. B 619 (2001) 709 [hep-ph/0011335] [INSPIRE].

[10] M. Pospelov and A. Ritz, Higgs decays to dark matter: beyond the minimal model, Phys. Rev. D 84 (2011) 113001 [arXiv:1109.4872] [INSPIRE].

[11] J. McDonald, Gauge singlet scalars as cold dark matter, Phys. Rev. D 50 (1994) 3637 [hep-ph/0702143] [INSPIRE].

[12] B. Patt and F. Wilczek, Higgs-field portal into hidden sectors, hep-ph/0605188 [INSPIRE].

[13] Y. Mambrini, Higgs searches and singlet scalar dark matter: Combined constraints from XENON 100 and the LHC, Phys. Rev. D 84 (2011) 115017 [arXiv:1108.0671] [InSPIRE].

[14] M. Raidal and A. Strumia, Hints for a non-standard Higgs boson from the LHC, Phys. Rev. D 84 (2011) 077701 [arXiv:1108.4903] [INSPIRE].

[15] X.-G. He and J. Tandean, Hidden Higgs boson at the LHC and light dark matter searches, Phys. Rev. D 84 (2011) 075018 [arXiv:1109.1277] [INSPIRE].

[16] E. Weihs and J. Zurita, Dark Higgs models at the 7 TeV LHC, JHEP 02 (2012) 041 [arXiv:1110.5909] [INSPIRE].

[17] U. Ellwanger, C. Hugonie and A.M. Teixeira, The next-to-minimal supersymmetric standard model, Phys. Rept. 496 (2010) 1 [arXiv:0910.1785] [INSPIRE].

[18] S. Chang, R. Dermisek, J.F. Gunion and N. Weiner, Nonstandard Higgs boson decays, Ann. Rev. Nucl. Part. Sci. 58 (2008) 75 [arXiv:0801.4554] [InSPIRE].

[19] B. Bellazzini, C. Csáki, J. Hubisz, J. Shao and P. Tanedo, Goldstone fermion dark matter, JHEP 09 (2011) 035 [arXiv:1106.2162] [INSPIRE].

[20] D. Bertolini, K. Rehermann and J. Thaler, Visible supersymmetry breaking and an invisible Higgs, JHEP 04 (2012) 130 [arXiv:1111.0628] [INSPIRE].

[21] C. Cheung and Y. Nomura, Higgs descendants, arXiv:1112.3043 [INSPIRE].

[22] B. Bellazzini, C. Csáki, A. Falkowski and A. Weiler, Buried Higgs, Phys. Rev. D 80 (2009) 075008 [arXiv:0906.3026] [INSPIRE].

[23] A. Falkowski, D. Krohn, L.-T. Wang, J. Shelton and A. Thalapillil, Unburied Higgs boson: Jet substructure techniques for searching for Higgs' decay into gluons, Phys. Rev. D 84 (2011) 074022 [arXiv: 1006.1650] [INSPIRE].

[24] I. Low, P. Schwaller, G. Shaughnessy and C.E. Wagner, The dark side of the Higgs boson, Phys. Rev. D 85 (2012) 015009 [arXiv:1110.4405] [INSPIRE].

[25] LeP Higgs Working for Higgs boson searches, AlEPH, DELPHi, CERN-L3, OPAL collaboration, Searches for invisible Higgs bosons: preliminary combined results using LEP data collected at energies up to $209 \mathrm{GeV}$, hep-ex/0107032 [INSPIRE].

[26] S. Frederiksen, N. Johnson, G.L. Kane and J. Reid, Detecting invisible Higgs bosons at the CERN Large Hadron Collider, Phys. Rev. D 50 (1994) 4244 [INSPIRE].

[27] O.J. Eboli and D. Zeppenfeld, Observing an invisible Higgs boson, Phys. Lett. B 495 (2000) 147 [hep-ph/0009158] [INSPIRE]. 
[28] R. Godbole, M. Guchait, K. Mazumdar, S. Moretti and D. Roy, Search for 'invisible' Higgs signals at LHC via associated production with gauge bosons, Phys. Lett. B 571 (2003) 184 [hep-ph/0304137] [INSPIRE].

[29] H. Davoudiasl, T. Han and H.E. Logan, Discovering an invisibly decaying Higgs at hadron colliders, Phys. Rev. D 71 (2005) 115007 [hep-ph/0412269] [INSPIRE].

[30] S.-h. Zhu, Detecting an invisibly Higgs boson at Fermilab Tevatron and CERN LHC, Eur. Phys. J. C 47 (2006) 833 [hep-ph/0512055] [InSPIRE].

[31] A. Dedes, T. Figy, S. Hoche, F. Krauss and T.E. Underwood, Searching for Nambu-Goldstone bosons at the LHC, JHEP 11 (2008) 036 [arXiv:0807.4666] [INSPIRE].

[32] S. Gopalakrishna, S.J. Lee and J.D. Wells, Dark matter and Higgs boson collider implications of fermions in an abelian-gauged hidden sector, Phys. Lett. B 680 (2009) 88 [arXiv: 0904.2007] [INSPIRE].

[33] ATLAS collaboration, Sensitivity to an invisibly decaying Higgs boson, PHYS-PUB-2009-061 (2009).

[34] CMS Collaboration, Sensitivity to an invisibly decaying Higgs boson, CMS-AN-2008-083.

[35] D. Cavalli et al., The Higgs working group: summary report, hep-ph/0203056 [INSPIRE].

[36] CMS collaboration, Search for new physics with a monojet and missing transverse energy in pp collisions at $\sqrt{s}=7$ TeV, PAS-EXO-11-059 (2011).

[37] ATLAS collaboration, G. Aad et al., Search for new phenomena with the monojet and missing transverse momentum signature using the ATLAS detector in $\sqrt{s}=7 \mathrm{TeV}$ proton-proton collisions, Phys. Lett. B 705 (2011) 294 [arXiv:1106.5327] [INSPIRE].

[38] ATLAS collaboration, Search for new phenomena in monojet plus missing transverse momentum final states using $1 \mathrm{fb}^{-1}$ of pp collisions at $\sqrt{s}=7 \mathrm{TeV}$ with the ATLAS Detector, ATLAS-CONF-2011-096 (2011).

[39] ATLAS collaboration, Search for a standard model Higgs in the $H \rightarrow Z Z \rightarrow$ llvv decay channel with 2.05 $\mathrm{fb}^{-1}$ of ATLAS data, ATLAS-CONF-2011-148 (2011).

[40] CMS collaboration, Search for the Higgs boson in the $H \rightarrow Z Z \rightarrow 2 \ell 2 \nu$ channel in $p p$ collisions at $\sqrt{s}=7 \mathrm{TeV}$, CMS-PAS-HIG-11-026 (2011).

[41] O. Lebedev, H.M. Lee and Y. Mambrini, Vector Higgs-portal dark matter and the invisible Higgs, Phys. Lett. B 707 (2012) 570 [arXiv:1111.4482] [INSPIRE].

[42] A. Djouadi, The anatomy of electro-weak symmetry breaking. I: the Higgs boson in the standard model, Phys. Rept. 457 (2008) 1 [hep-ph/0503172] [INSPIRE].

[43] N.D. Christensen and C. Duhr, FeynRules - Feynman rules made easy, Comput. Phys. Commun. 180 (2009) 1614 [arXiv:0806.4194] [INSPIRE].

[44] T. Sjöstrand, S. Mrenna and P.Z. Skands, PYTHIA 6.4 physics and manual, JHEP 05 (2006) 026 [hep-ph/0603175] [INSPIRE].

[45] J.S. Conway, Pretty good simulation of high-energy collisions, 090401 release.

[46] M.L. Mangano, M. Moretti, F. Piccinini and M. Treccani, Matching matrix elements and shower evolution for top-quark production in hadronic collisions, JHEP 01 (2007) 013 [hep-ph/0611129] [INSPIRE].

[47] J. Baglio and A. Djouadi, Higgs production at the LHC, JHEP 03 (2011) 055 [arXiv: 1012.0530] [INSPIRE].

[48] C. Englert, J. Jaeckel, E. Re and M. Spannowsky, Evasive Higgs maneuvers at the LHC, Phys. Rev. D 85 (2012) 035008 [arXiv:1111.1719] [INSPIRE]. 
[49] J. Gallicchio and M.D. Schwartz, Quark and gluon tagging at the LHC, Phys. Rev. Lett. 107 (2011) 172001 [arXiv:1106.3076] [INSPIRE].

[50] ATLAS collaboration, G. Aad et al., Search for a heavy gauge boson decaying to a charged lepton and a neutrino in $1 \mathrm{fb}^{-1}$ of pp collisions at $\sqrt{s}=7 \mathrm{TeV}$ using the ATLAS detector, Phys. Lett. B 705 (2011) 28 [arXiv:1108.1316] [INSPIRE].

[51] P Gagnon, Invisible Higgs boson decays in the ZH and WH channels, PHYS-PUB-2005-011 (2005).

[52] V. Del Duca, W. Kilgore, C. Oleari, C. Schmidt and D. Zeppenfeld, Gluon fusion contributions to $H+2$ jet production, Nucl. Phys. B 616 (2001) 367 [hep-ph/0108030] [INSPIRE].

[53] A. Nikitenko and M.L. Vazquez Acosta, Monte Carlo study of $g g \rightarrow H+$ jets contribution to vector boson fusion Higgs production at the LHC, arXiv:0705.3585 [INSPIRE].

[54] B.E. Cox, J.R. Forshaw and A.D. Pilkington, Extracting Higgs boson couplings using a jet veto, Phys. Lett. B 696 (2011) 87 [arXiv:1006. 0986] [INSPIRE].

[55] LhC Higgs Cross Section Working Group collaboration, S. Dittmaier et al., Handbook of LHC Higgs cross sections: 1. Inclusive observables, arXiv:1101.0593 [INSPIRE].

[56] T. Figy, S. Palmer and G. Weiglein, Higgs production via weak boson fusion in the standard model and the MSSM, JHEP 02 (2012) 105 [arXiv:1012.4789] [INSPIRE].

[57] K. Arnold et al., VBFNLO: a parton level Monte Carlo for processes with electroweak bosons - Manual for version 2.5.0, arXiv:1107.4038 [INSPIRE].

[58] C. Berger et al., Precise predictions for $W+4$ jet production at the Large Hadron Collider, Phys. Rev. Lett. 106 (2011) 092001 [arXiv: 1009.2338] [INSPIRE].

[59] H. Ita et al., Precise predictions for $Z+4$ jets at hadron colliders, Phys. Rev. D 85 (2012) 031501 [arXiv: 1108.2229 ] [INSPIRE].

[60] CMS collaboration, Data-driven estimation of the invisible Z background to the SUSY MET plus jets search, PAS-SUS-08-002 (2008).

[61] CMS collaboration, Search for new physics at CMS with jets and missing momentum, PAS-SUS-10-005 (2010).

[62] Z. Bern et al., Driving missing data at next-to-leading order, Phys. Rev. D 84 (2011) 114002 [arXiv:1106.1423] [INSPIRE].

[63] C. Oleari and D. Zeppenfeld, QCD corrections to electroweak $\nu(l) j j$ and $\ell^{+} \ell^{-} j j$ production, Phys. Rev. D 69 (2004) 093004 [hep-ph/0310156] [INSPIRE].

[64] K. Kondo, Dynamical likelihood method for reconstruction of events with missing momentum. 1: method and toy models, J. Phys. Soc. Jpn. 57 (1988) 4126.

[65] D0 collaboration, V. Abazov et al., A precision measurement of the mass of the top quark, Nature 429 (2004) 638 [hep-ex/0406031] [inSPIRE].

[66] ATLAS collaboration, ATLAS sensitivity prospects for 1 Higgs boson production at the LHC Running at 7, 8 or 9 TeV, PHYS-PUB-2010-015 (2010).

[67] CMS collaboration, The CMS physics reach for searches at $7 \mathrm{TeV}$, CMS-NOTE-2010-008 (2010). 